\title{
Mit wem über Religion sprechen? Kirchenbindung und Opportunitäten als Determinanten der Wahl von Gesprächspartnern
}

\author{
von \\ Heiner Meulemann
}

\begin{abstract}
Untersucht wird, wie die Häufigkeit von Gesprächen über Religion von der Kirchenbindung und von Opportunitäten abhängt. Am häufigsten sollte mit Familienmitgliedern, weniger häufig mit kirchlichen Professionellen und am seltensten mit Personen jenseits der Familie gesprochen werden - Partikularismushypothese. Kirchennahe sollten mit kirchlichen Professionellen und Familienmitgliedern häufiger als Kirchenferne, mit Personen jenseits der Familie aber gleich häufig sprechen - Zuständigkeits- und Bedarfshypothese. Ehe und Elternschaft sollten Gespräche mit kirchlichen Professionellen und Familienmitgliedern, nicht aber mit Personen jenseits der Familie steigern - Lebenslaufhypothese. Vertrauen und Sozialstatus sollten Gespräche mit kirchlichen Professionellen und Familienmitglieder nicht, wohl aber mit Personen jenseits der Familie steigern - Ressourcenhypothese. Diese Hypothesen werden im ALLBUS 2012 unter denen geprüft, die überhaupt über Religion sprechen. Die Partikularismushypothese wird nicht, die Zuständigkeits- und Bedarfshypothese sowie die Lebenslauf- und Ressourcenhypothese werden tendenziell bestätigt.
\end{abstract}

Stichworte: Gespräche über Religion, Kirchenbindung, christliches und naturalistisches Weltbild, kirchliche Professionelle.

Weil jeder weiß, dass er sterben muss, kann niemand der Frage entkommen, wozu er auf der Welt ist. Sie ist nicht umsonst die Frage Nr. 1 im Katechismus der Konfessionen. Sie kann als das Bezugsproblem der Religion (Hahn, 1974, S. 14, 39-43, 86-106), als der „universelle Aspekt“ der Beziehung zwischen Mensch und Religion (Stark, 1996, S. 36; Stark \& Bainbridge, 1987, S. 44, 52) gesehen und die religiöse Frage genannt werden. Aber Antwort gibt immer eine bestimmte Religion, in Deutschland also immer noch überwiegend das Christentum. Weder die religiöse Frage noch die Religion überhaupt, wohl aber die Lehren und Praktiken des Christentums trennen zwischen Gläubigen und Ungläubigen. Die religiöse Frage stellt sich Gläubigen und Ungläubigen in gleicher Weise; aber „die Religion“ ist eine Abstraktion, die Gläubigen und Ungläubigen von ihren unterschiedlichen Ausgangspunkten zum Thema werden kann.

Die Beschäftigung mit Religion steht daher zwischen der Beschäftigung mit der religiösen Frage und mit der christlichen Antwort. Sie richtet sich auf eine gedanklich konstruierte Lebenssphäre, die in der menschlichen Situation begründet ist und sich in der jeweiligen Kultur konkretisiert. Sie führt von der unumgänglichen Frage zur breiten Palette der möglichen Antworten. Sie entdeckt Möglichkeiten, die im Blick auf die Frage noch nicht sichtbar waren und mit der Übernahme einer Antwort wieder aus dem Blick genommen werden können. Entsprechend führen zwei Wege dazu, sich mit Religion zu beschäftigen. Einerseits muss, wer nicht fest in einem Glauben lebt, unter den Möglichkeiten eine Antwort auf die religiöse Frage suchen. Anderseits kann auch der Gläubige an seinem Glauben zweifeln und unsicher werden, so dass er von Überzeugungen zu Möglichkeiten zurückkehrt. Wer glaubt und praktiziert, also kirchennah ist, sollte sich also auf andere Weise mit der Religion beschäftigen als 
wer nicht glaubt und praktiziert, also kirchenfern ist. Die Kirchenbindung ist der erste Bestimmungsfaktor für die Beschäftigung mit Religion.

Mit Religion kann man sich individuell und im Gespräch mit anderen beschäftigen. Wenn Religion Kommunikation über die religiöse Frage, über die Grenze zwischen Immanenz und Transzendenz ist (Tyrell, Krech \& Knoblauch,1998, S. 17; Sammet, 2006, S. 358-371; Nassehi, 2009, S. 192-196), dann ist das Gespräch - die Kommunikation unter Anwesenden - ein wichtiges Element der Religiosität, also der persönlich praktizierten Religion. Als Partner sind die Vertreter der Kirchen zuständig, aber nicht so leicht erreichbar wie Familienmitglieder und vielleicht auch Berufskollegen, Nachbarn und Freunde. Die Wahl eines Partners hängt also von der Kirchenbindung, aber wie bei jedem Gespräch auch von den Opportunitäten ab. Die Frage ist also, ob, wer überhaupt über Religion spricht, unterschiedliche Partner in unterschiedlicher Weise nach Kirchenbindung und Opportunitäten wählt. Sie wird im Folgenden untersucht. In Abschnitt 1 werden Hypothesen, Daten und Variablen, in Abschnitt 2 und 3 bivariate und multivariate Ergebnisse dargestellt.

\section{Untersuchungsanlage}

\subsection{Hypothesen}

Partikularismus: Rangfolge der Häufigkeiten

Religion ist ein privates Thema. Wie häufig man darüber mit anderen spricht, hängt zunächst von der Enge der Beziehung zu ihnen ab. Was man umgangssprachlich als private oder persönliche, enge oder intime Beziehungen bezeichnet, lässt sich mit dem Begriff des Partikularismus erfassen. Partikularistische Beziehungen sind durch die Personen und nicht durch eine Kategorie bestimmt (Parsons \& Shils, 1951). Prototypisch ist hier die Familie. Es gibt viele Väter, aber nur mein Vater ist "mein Vater“; und der Tod meines Vaters ist ein „unersetzlicher Verlust“. Universalistische Beziehungen hingegen sind durch eine Kategorie und nicht durch die Personen bestimmt. Kollegen definieren sich durch die Berufszugehörigkeit, Nachbarn durch die Nähe, Geschäftspartner durch den Tausch, Mitbürger durch die Nationalität, Vereinsfreunde durch die Mitgliedschaft. Die Personen sind austauschbar; „niemand ist unersetzlich“.

Mit Mitgliedern der eigenen Familie lebt man - räumlich und psychisch - so eng zusammen, wie es der Verwandtschaftsgrad fordert. Trotz unterschiedlicher Verwandtschaftsgrade, Haushaltsformen und Wohndistanzen schafft der Partikularismus der Familie ohne besondere Anstrengungen der Beteiligten Vertrautheit und Nähe, die Gespräche über jedes Thema erleichtern - insbesondere aber über ein persönliches Thema wie Religion. Empirisch wurde 2002 über Religion unter Evangelischen wie unter Konfessionslosen am häufigsten mit Familienmitgliedern gesprochen (Huber, Friedrich \& Steinacker, 2006, S. 462). In einer Befragung über die Wichtigkeit von Lebensbereichen 2012 auf sieben Stufen (eigene Auswertung des ALLBUS) liegt die Wichtigkeit der Familie mit 6,5 ganz oben und fast einen Skalenpunkt vor den gleich eingeschätzten Bereichen Beruf, Freizeit und Freunde. Mit Familienmitgliedern sollte man daher am häufigsten über Religion sprechen.

Außerhalb der Familie bewegt man sich hingegen meist in universalistischen Beziehungen. Unter Berufskollegen, Geschäftspartnern, Mitbürgern und Nachbarn entsteht daher im Regelfall und ohne persönliche Anstrengung nicht die Vertrautheit, die ein Gespräch über Themen jenseits der Beziehung und insbesondere über Religion erleichtert. Empirisch wurde 2002 über Religion unter Evangelischen wie unter Konfes- 
sionslosen mit Freunden und Bekannten, Arbeitskollegen und Nachbarn seltener als mit Familienmitgliedern gesprochen (Huber et al., 2006, S. 462). Mit Personen jenseits der Familie sollte man daher seltener als mit Familienmitgliedern über Religion sprechen.

Einige universalistische Beziehungen können sich jedoch auch partikularistischen annähern. Freunde teilen Interessen, aber keine Intimität; Mannschaftskameraden und Kegelbrüder sind leicht austauschbar. Aber je mehr zwei Freunde sich über ihre persönlichen Angelegenheiten austauschen, je „enger" die Freundschaft wird, desto mehr nähert sie sich einer partikularistischen Beziehung an. Am Ende ist nur mein Freund „mein“ Freund. Die Freundschaft ist potenziell partikularistisch. Was für die frei gestaltbare Beziehung Freundschaft gilt, gilt auch für viele zur berufsmäßigen Hilfe hin vordefinierten Beziehungen: Beratung oder Heilung, Fürsorge oder Seelsorge. Je mehr ich mich meinem Helfer offenbart habe und je mehr er mir geraten hat, desto schwerer wird es, die Personen auszutauschen, ohne das Ziel der Beziehung zu verfehlen. Am Ende ist nur mein Therapeut „mein Therapeut“, und nur mein Pfarrer "mein Pfarrer".

Der potenzielle Partikularismus ist Resultat eines Prozesses der Annäherung. Im hier thematischen Fall des kirchlichen Professionellen wird die Annäherung durch die Kirchenbindung des Gesprächssuchenden ausgelöst und verstärkt. Kirchennahe haben mit kirchlichen Professionellen durch Glauben und Ritus mehr Gemeinsamkeiten als Kirchenferne. Je enger jemand der Kirche verbunden ist, desto weniger sieht er seinen Pfarrer als Mitglied einer universalistischen Gruppe, desto mehr bringt er partikularistische Elemente in die Beziehung und nähert sie einer Familienbeziehung an. ${ }^{1}$ Für Kirchenferne hingegen bleibt die Beziehung zu kirchlichen Professionellen so universalistisch wie zu anderen Personen jenseits der Familie. Zwar bleiben auch in einer kirchennahen Familie die kirchlichen Professionellen bei aller Vertrautheit Spezialisten. Für Kirchenferne aber verlieren sie sich in die Anonymität der Gesellschaft; Pfarrer sind eine Berufsgruppe wie jede andere. Empirisch wurde 2002 über Religion mit Pfarrern unter Evangelischen häufiger, und unter Konfessionslosen seltener als mit Arbeitskollegen und Nachbarn gesprochen (Huber et al., 2006, S. 462). Mit kirchlichen Professionellen sollte man daher seltener als mit Familienmitgliedern, aber häufiger als mit Personen jenseits der Familie über Religion sprechen. Das bezeichnen wir als Partikularismushypothese.

Der Partikularismus der Beziehung zu Familienmitgliedern, der potenzielle Partikularismus der Beziehung zu kirchlichen Professionellen und der Universalismus der Beziehung zu Personen jenseits der Familie bestimmen also die Rangfolge der Gesprächshäufigkeit. Zugleich kann die Kirchenbindung des Gesprächssuchenden den potenziellen Partikularismus der Beziehung zu kirchlichen Professionellen zu Tage bringen. Worauf beruht der Einfluss der Kirchenbindung auf die Gesprächshäufigkeit mit kirchlichen Professionellen? Und welchen Einfluss übt die Kirchenbindung auf die Gesprächshäufigkeit mit Familienmitgliedern und Personen jenseits der Familie aus?

1 Der Katholizismus erfasst den potenziellen Partikularismus der Beziehung zwischen Priester und Laien auch terminologisch. Der Beichtvater kann zu seinem Beichtkind, das ihm wiederholt sein Leben erzählt, eine persönliche Beziehung aufbauen, in der keine Seite ohne weiteres ersetzt werden kann. Ob das so sein soll und sein kann, ist allerdings sowohl theologisch wie psychologisch eine Frage (Ziemann, 2007, S. 274-290). 


\section{Kirchenbindung: Zuständigkeit und Bedarf}

Zuständig für ein Gespräch über Religion sind die kirchlichen Professionellen. Wer als Theologe ausgebildet ist und im geistlichen Beruf arbeitet, hat die Kompetenz, auf Fragen der Religion und des Glaubens eine Antwort zu geben. Wer im kirchlichen Gesundheits-, Sozial- und Bildungswesen arbeitet, hat - sofern es nicht um Gesundheit, Soziales oder Bildung, sondern um Religion geht - dieselbe Kompetenz mit schwächerer Autorität und muss Gesprächssuchende im Zweifelsfall an Geistliche weiterleiten. Den kirchlichen Professionellen stehen Kirchennahe wie Kirchenferne als religiöse Laien ohne Kompetenz (Karle, 2001; Krech \& Höhmann, 2005, S. 204; Schützeichel, 2010, S. 128-140; Ziemann, 2009: S. 96-112) gegenüber - so wie den Medizinern die medizinischen oder den Juristen die juristischen Laien. Anders aber als medizinische oder juristische Laien wenden sich religiöse Laien an ihre Professionellen nicht nur nach vermuteter Kompetenz oder empfundener Sympathie, sondern auch nach Zugehörigkeit; eben deshalb ist es treffender, ihre Partner als kirchliche statt als religiöse Professionelle zu bezeichnen. Nur Mitgliedern der gleichen Kirche und Anhängern des gleichen Glaubens, also Kirchennahen ist die Zuständigkeit "ihrer" kirchlichen Professionellen selbstverständlich. Kirchenferne müssen die Zuständigkeit zuerst anerkennen, also eine Barriere überwinden. Empirisch gaben 2002 Evangelische weitaus häufiger als Konfessionslose an, mit Pfarrern über Religion zu sprechen (Huber et al., 2006, S. 462). Daraus ergibt sich die Zuständigkeitshypothese: Mit kirchlichen Professionellen sollten Kirchennahe häufiger als Kirchenferne über Religion sprechen.

Wenn nicht mit kirchlichen Professionellen, so sprechen religiöse Laien untereinander über Religion. Dann ist die Wahl nicht mehr durch die Zuständigkeit, sondern durch den Bedarf bestimmt. Den Bedarf an religiösen Gesprächen sucht man zuerst mit Mitgliedern seiner Familie zu befriedigen.

Familienmitglieder teilen - idealerweise, und oft auch faktisch - konfessionelle Zugehörigkeit, christliche oder säkulare Glaubensüberzeugungen, kirchliche Riten wie Gottesdienstbesuch oder Gebet und Wendepunkte des Lebens, die in den kirchlichen Kasualien oder säkularen Feiern begangen werden. Da in Gesamtdeutschland 2012 etwa 80 \% der Bevölkerung Mitglieder einer Kirche sind (Meulemann, 2013, S. 346), bringen kirchliche Riten und Wendepunkte des Lebens die Familie als Partnerin der kirchlichen Professionellen zusammen. Sie machen die Familie zu einem zweiten Forum der Religion nach der Kirche. Die Familienmitglieder sollten daher miteinander über ihren religiösen Bedarf (Krech \& Höhmann, 2005, S. 209-211) sprechen, bevor und während sie mit kirchlichen Professionellen zusammenkommen. Glaubensprobleme diskutieren Ehegatten oder Eltern und Kinder unter sich, bevor sie sich an den Pfarrer wenden; Taufen, Hochzeiten und Beerdigungen plant die Familie mit dem Pfarrer. Familienmitglieder haben also, wenn sie kirchennah sind, einen größeren Bedarf über Religion zu sprechen, als wenn sie kirchenfern sind. Gewiss haben auch Kirchenferne Bedarf, über weltanschauliche Überzeugungen und Wendepunkte des Lebens miteinander zu sprechen; aber sie sind nicht gehalten, sich gegenüber kirchlichen Professionellen gemeinsam zu erklären.

Personen jenseits der Familie teilen hingegen diesen Bedarf nicht. Mit Berufskollegen spricht man über die Arbeit, mit Freunden über die Freizeit, mit Nachbarn über die Nachbarschaft. Mit allen kann man auch über Religion reden - ebenso wie über Politik, Kultur oder Wirtschaft. Aber der Bedarf dazu ist der Beziehung nicht einprogrammiert; er findet sich nach Interesse und Gelegenheit, die zwischen Kirchennahen und Kirchenfernen nicht diskriminieren. 
Aus diesen Überlegungen ergeben sich die positive und negative Seite der Bedarfshypothese: Mit Familienmitgliedern sollten Kirchennahe häufiger als Kirchenferne über Religion sprechen. Und: Mit Personen jenseits der Familie sollten Kirchennahe und Kirchenferne gleich häufig über Religion sprechen.

\section{Grafische Darstellung}

Die Partikularismus-, Zuständigkeits- und Bedarfshypothese sind in Abbildung 1 zusammengefasst. Die Partikularismushypothese sagt eine Rangfolge der Häufigkeiten „Familie, kirchliche Professionelle, Personen jenseits der Familie“ in der gesamten Bevölkerung, also im Mittel von Kirchennahen und Kirchenfernen voraus. Bei Kirchenfernen sollten die Häufigkeiten für kirchliche Professionelle und Personen jenseits der Familie zusammenfallen. Die Zuständigkeit der kirchlichen Professionellen und der positive Bedarf der Familie äußern sich im Anstieg der Häufigkeiten mit der Kirchennähe, der negative Bedarf der Personen jenseits der Familie in der Unabhängigkeit der Häufigkeit von der Kirchennähe.

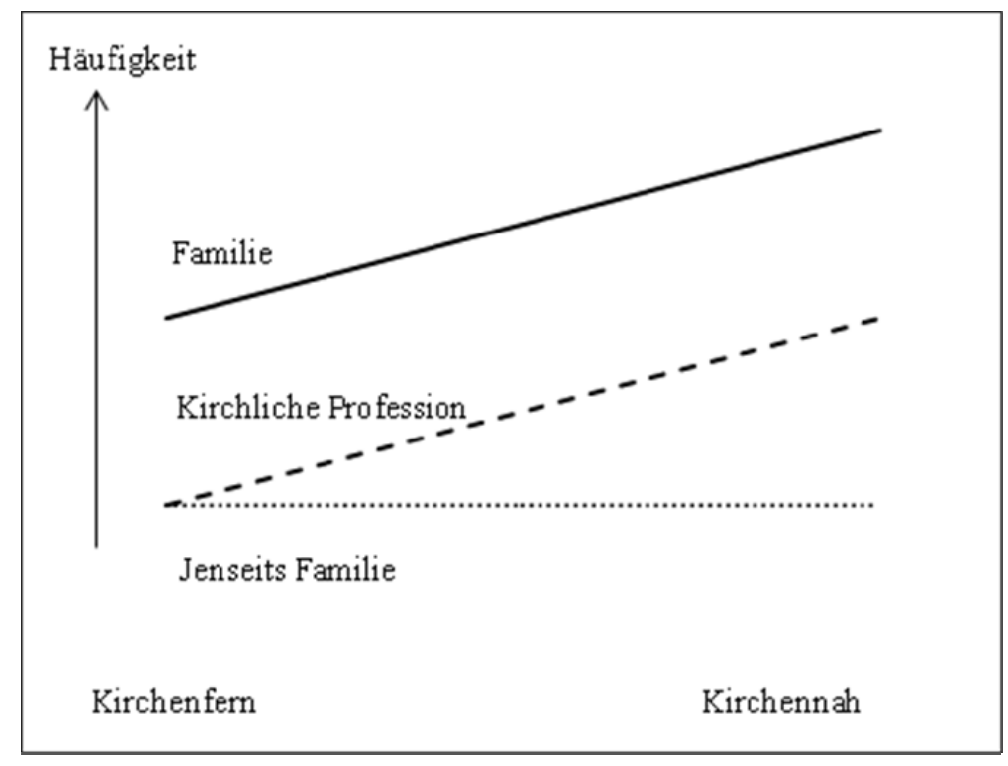

Abbildung 1. Partikularismus- sowie Zuständigkeits- und Bedarfshypothese: Vorausgesagte Häufigkeiten des Gesprächs über Religion mit kirchlichen Professionellen, Familienmitgliedern und Personen jenseits der Familie bei Kirchennahen und Kirchenfernen.

Die in Abbildung 1 zusammengefassten Hypothesen sollten für beide Konfessionen gelten, aber Unterschiede zwischen den Konfessionen sind schwer voraussagbar. Einerseits betont der Protestantismus die Innerlichkeit und Individualität des Glaubens von Priestern und Laien, der Katholizismus das Amtscharisma des Priesters in der kirchlichen Hierarchie und die „Orthopraxie“ der Riten (Ziemann, 2009 S. 59-60, 102-107), so dass das Gespräch mit den Gläubigen über Religion für den protestantischen Pfarrer in stärkerem Maße Teil der alltäglichen gemeindlichen Arbeit als für den katholischen Pfarrer sein sollte. Zudem bietet Luthers Lehre vom Priesterstand aller Gläubigen die Rechtfertigung für Gespräche der Laien mit kirchlichen Professionellen über Religion. Anderseits hat die katholische Kirche das Gespräch über die kirchlich korrekte Lebensführung im Sakrament der Beichte geregelt und zur jährlichen Pflicht erklärt (Ziemann, 2007, S. 274-290). Wie diese Überlegungen zeigen, motiviert nicht die Konfessionszugehörigkeit an sich zum Gespräch über Religion, sondern die Aktivität des Mitglieds in seiner Kirche, also die Kirchenbindung. Wenn 
die Kirchenbindung kontrolliert ist, sollte die Konfessionsmitgliedschaft keinen - oder nur einen schwachen - Einfluss auf die Wahl eines Gesprächspartners haben.

Der Partikularismus der Beziehung zum Gesprächspartner und die Kirchenbindung des Gesprächssuchenden sind also die besonderen, im Thema liegenden Bestimmungsgründe für ein Gespräch über Religion. Aber wie jedes Gespräch hängt auch das über Religion von Opportunitäten $a b$, die im privaten Lebenslauf und mit den Ressourcen des Gesprächssuchenden variieren.

\section{Opportunitäten: Privater Lebenslauf und Ressourcen}

Wenn die Wendepunkte des Lebens kirchliche Professionelle und Familien verstärkt zusammenführen, dann sollte der Status einer Person im privaten Lebenslauf Anlass sein, mit kirchlichen Professionellen und mit Familienangehörigen über Religion zu sprechen. Partner- und Elternschaft sind Voraussetzung für Gespräche über Religion mit Partnern und Kindern und verlangen Gespräche über Religion mit ihnen und mit kirchlichen Professionellen, sobald Übergänge anstehen und auch noch wenn sie vollzogen sind. Aber sie legen es nicht nahe, mit Personen jenseits der Familie zu sprechen. Daraus ergibt sich die Lebenslaufhypothese: Mit kirchlichen Professionellen und Familienangehörigen sollten Verheiratete und Eltern über Religion häufiger als Personen eines anderen Familienstands sprechen - mit Personen jenseits der Familie jedoch gleich häufig.

Der Status im privaten Lebenslauf variiert mit Alter und Geschlecht. Um seine eigenständigen Einflüsse zu erfassen, müssen Alter und Geschlecht kontrolliert werden. Und weil die privaten Verpflichtungen sich in der Lebensmitte kumulieren, muss das Alter als lineare und quadrierte Variable kontrolliert werden.

Religion ist eine persönliche Angelegenheit, über die man in der Familie und in der Kirche, aber nicht ohne weiteres in der Öffentlichkeit spricht. Wer jemanden jenseits von Familie oder Kirche darüber anspricht, muss etwas von sich selber preisgeben und kann auf Missachtung stoßen. Er sollte deshalb Vertrauen zu dem anderen haben, den er nicht näher kennt und dessen Reaktion ungewiss ist. Vertrauen in Personen jenseits der Familie ist eine emotionale Ressource für ein Gespräch mit ihnen. Familienmitglieder - und idealiter auch kirchliche Professionelle - kennt man, einerlei wie man sie einschätzt. Vertrauen ist nicht erforderlich, um die Distanz zu überwinden und ein Gespräch einzuleiten. Wer jemanden jenseits von Familie und Kirche über Religion ansprechen will, muss sich weiterhin in der Öffentlichkeit artikulieren und Ansprechpartner mobilisieren können. Nicht nur politisches Engagement, sondern jede öffentliche Stellungnahme wird durch ein „inneres Selbstwirksamkeitsgefühl“ (zuletzt dazu Gabriel, 2013, S. 167-170) gefördert. Das Gespräch über Religion jenseits von Familie oder Kirche verlangt also kognitive und soziale Ressourcen, die für ein Gespräch mit Familienangehörigen und kirchlichen Professionellen nicht gebraucht werden. Daraus ergibt sich die Ressourcenhypothese: Mit Personen jenseits der Familie sollte jemand umso häufiger über Religion sprechen, je mehr emotionale, kognitive und soziale Ressourcen er hat - mit kirchlichen Professionellen und Familienangehörigen jedoch unabhängig von Ressourcen.

\subsection{Datensatz und Zielvariable}

Die Untersuchungsdaten stammen aus dem ALLBUS 2012, der von der Deutschen Forschungsgemeinschaft finanziert und der Wissenschaftlergemeinde kostenlos zur Verfügung steht (http://www.gesis.org/allbus/studienprofile/2012/). Er wird für Gesamtdeutschland mit Gewichtung nach der Bevölkerungsgröße der beiden Landestei- 
le analysiert. Weil das Gespräch mit kirchlichen Professionellen der zentrale Untersuchungsgegenstand ist, wurden alle Personen mit nicht christlichen Religionszugehörigkeiten - 3,8 \% der Stichprobe - ausgeschlossen. Personen ohne Religionszugehörigkeit - 26,9 \% der Stichprobe - wurden jedoch in der Stichprobe belassen, weil sie durch die christliche Herkunft geprägt waren, aber sich von ihr distanziert hatten.

Gefragt wurde „Wie oft unterhalten Sie sich mit anderen Menschen über religiöse Themen?" mit fünf Intensitätsstufen von „Nie“(1), „Selten“, „Manchmal“, "Oft" bis „Sehr oft" (5). ${ }^{2}$ Wer wenigstens „selten“ gewählt hatte, wurde weiter gefragt: „Und mit wem unterhalten Sie sich zumindest gelegentlich über religiöse Themen?"Weil die Häufigkeit in einer Regression stark positiv von der Kirchgangshäufigkeit (beta=.41) und schwach negativ von der religiösen Indifferenz (beta=-.15) abhängt und positiv mit dem christlichen Weltbild $(r=.38)$ und figurativen wie konzeptuellen Glaubensüberzeugungen ( $r=.29$ und .32) sowie negativ mit dem naturalistischen Weltbild ( $r=-$ .22) korreliert, rekrutiert sich die Gruppe derer, die einen Gesprächspartner genannt haben, im Schwerpunkt stärker als die Gesamtstichprobe aus kirchennahen Personen. Sie umfasst 2750 Personen, 82,3 \% der Gesamtstichprobe. Wenn man das Gespräch über Religion als Indiz nimmt, ist das religiöse Bedürfnis in Deutschland 2012 keineswegs gering.

Die Gesprächspartner wurden aus folgender Liste gewählt: „A Mit meinen Eltern“, „B Mit meinem Ehe- bzw. Lebenspartner", „C Mit meinen Kindern“, „D Mit sonstigen Verwandten“, „E Mit Freunden“, „F Mit Bekannten, Nachbarn oder Arbeitskollegen“, „G Mit einem Geistlichen (Pfarrer, Iman, Rabbiner, o.ä.)“, „H Mit Menschen in kirchlichen oder anderen religiösen Gruppen“, „I Mit sonstigen Personen“. Mehrfachantworten waren möglich. Durchschnittlich wurden 2,65 Personen genannt. Die Vorgaben H und I wurden als kirchliche Professionelle, die Vorgaben A bis D als Familienmitglieder, und Vorgaben E, F und I als Personen jenseits der Familie zusammengefasst. Durchschnittlich wurden zu den 2, 4 und 3 Vorgaben dieser drei Klassen 0,35, 1,27, 1,03 Personen genannt.

Da die Häufigkeit der Nennungen mit der Zahl der Vorgaben steigt, wurden sie dichotomisiert: $26,8 \%$ nannten kirchliche Professionelle, 74,2 \% Familienmitglieder und 73,2 \% Personen jenseits der Familie. Diese Dichotomien sind die Zielvariablen der Analyse: KIRPROF, FAMILIE, JENFAM. Sie überlappen sich: 16,3 \% nannten Partner aller drei Gruppen, 4,7 \% kirchliche Professionelle und Familienangehörige, 3,9 \% kirchliche Professionelle und Personen jenseits der Familie, 32,9 \% Familienangehörige und Personen jenseits der Familie; rund ein Drittel nannten nur Partner aus einer der drei Gruppen. KIRPROF korreliert nur schwach mit FAMILIE und JENFAM ( $r=.06$ und .03); FAMILIE und JENFAM korrelieren etwas stärker negativ $(r=-.26)$.

2 Die Formulierung erlaubt es nicht, zwischen einem persönlich verbindlichen „religiösen Gespräch“ und einem unverbindlichen „Gespräch über Religion“ (Sammet, 2006, S. 374) zu unterscheiden. Da die Frage nach zahlreichen anderen Fragen zum Glauben und zur kirchlichen Praxis gestellt wurde, sollte sie im Sinne eines „religiösen Gesprächs“ verstanden worden sein. Aber auch die Gegenstände eines „religiösen Gesprächs“ bleiben unscharf: der wahre Glauben und die rechte Praxis gehören sicher für Gläubige und Ungläubige dazu. Aber ist die katholische Beichte eine sakramentale Interaktion, eine „Technik der Menschenführung“ (Ziemann, 2007, S. 275) oder ein therapeutisches Gespräch? Man kann vermuten, dass viele praktizierende Katholiken die Beichte nicht als Gespräch „über religiöse Themen“ auffassen und dass Protestanten einen Gottesdienst eher als Gespräch verstehen als Katholiken, dass also bereits die Frage „protestantisch“ ist. Leider sind die „religiösen Themen“ im ALLBUS nicht erfragt worden. 


\subsection{Prädiktorvariablen}

Kirchenbindung wird positiv durch die Kirchgangshäufigkeit KIRCHG auf sechs Stufen und durch das christliche Weltbild CHRI sowie negativ durch das naturalistische Weltbild NAT erhoben. CHRI und NAT wurde nach den Ergebnissen einer explorativen Faktorenanalyse (Meulemann, 2014b) des Weltanschauungs-Inventars von Felling, Peters und Schreuder (1987) gebildet, wobei CHRI auf theistischen und deistischen Aussagen beruhte. ${ }^{3}$ Die Kirchenbindung wird weiterhin - Praxis und Glauben zusammenfassend - positiv als die Wichtigkeit von „Religion und Kirche“ WRELIKIR auf sieben Stufen neben anderen Lebensbereichen wie Familie, Beruf, Freizeit und Politik erhoben. Weil das naturalistische Weltbild das säkulare Gegenstück zur christlich-kirchlichen Religion ist, sollten sich die Einflüsse umkehren.

Der private Lebenslauf wurde durch Kodiervariablen der Verheiratung und Elternschaft, EHE und KIND erhoben. Kontrolliert wurde das Alter in Jahren und als quadrierte Abweichung vom Mittel, ALTER und ALTERQU.

Der soziale Status wurde durch Bildung und Berufsprestige, BILDUNG und BPSIOPS erhoben. Vertrauen in Mitmenschen wurde so erfragt: „Manche Leute sagen, dass man den meisten Menschen trauen kann. Andere meinen, dass man nicht vorsichtig genug sein kann im Umgang mit anderen Menschen. Was ist Ihre Meinung dazu? (3) Den meisten Menschen kann man trauen, (1) Man kann nicht vorsichtig genug sein, (2) Das kommt darauf an" und mit den Werten in Klammern als VERTRAUEN verkodet.

Die Konfessionszugehörigkeit zur evangelischen oder katholischen Kirche wurde durch die Kodiervariablen EVAN und KATH mit der Basis der Konfessionslosigkeit, das Geschlecht mit der Kodiervariable FRAU und der Landesteil mit der Kodiervariable OST kontrolliert.

\section{Ergebnisse bivariat}

Die durchschnittlichen Personenzahlen und die Nennungen überhaupt ohne Kontrolle der Kirchenbindung in Abschnitt 1.2 haben bereits gezeigt, dass die Partikularismushypothese nicht bestätigt wird. Familienmitglieder und Personen jenseits der Familie werden gleich häufig und weit häufiger als kirchliche Professionelle genannt. Partikularistische und universalistische Beziehungen bilden also nicht wie erwartet die Extreme, sondern fallen zusammen; und eine universalistische Beziehung, in die man partikularistische Elemente einfügen kann, liegt nicht wie erwartet dazwischen, sondern am Ende. Die religiöse ist keine kirchliche, sondern gleichermaßen familiale wie außerfamiliale Kommunikation; Familie sowie Freunde, Nachbarn und Kollegen stehen im Zentrum, Geistliche am Rande.

\subsection{Kirchenbindung}

Die Kirchgangshäufigkeit hängt nicht nur in der gesamten Stichprobe mit der Häufigkeit der Diskussion über Religion zusammen ( $r=.49)$, sondern auch in der Gruppe derer, die überhaupt über Religion sprechen, mit der Zahl der genannten Gesprächspartner $(r=.40)$. Wie sich die Gesprächspartner aus kirchlichen Professionel-

3 Eine theistische Aussage war z.B. „Es gibt einen Gott, der sich mit jedem Menschen persönlich befasst"; eine deistische „Das Leben hat nur eine Bedeutung, weil es einen GOTT gibt"; eine naturalistische „Unser Leben wird letzten Endes bestimmt durch die Gesetze der Natur“. 
Ien, Familienangehörigen und Personen jenseits der Familie in Abhängigkeit von der Kirchgangshäufigkeit verteilen, stellt Abbildung 2 dar.

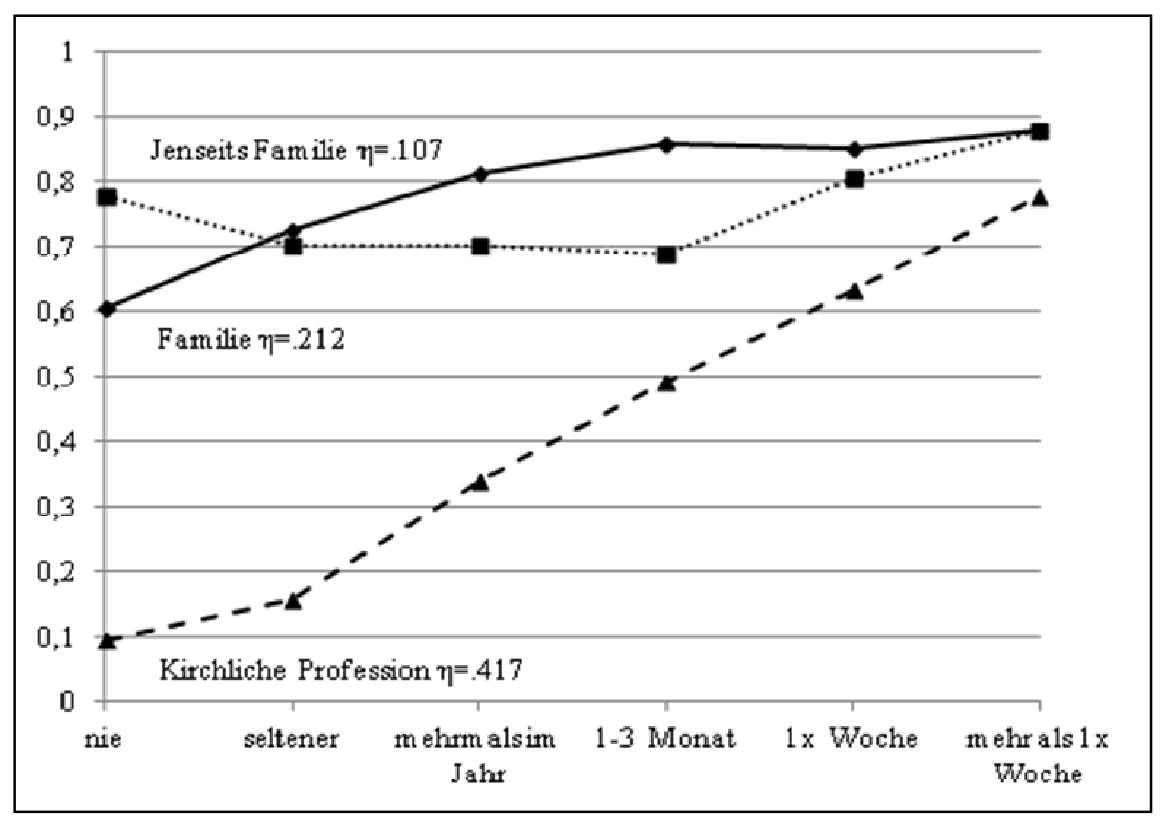

Abbildung 2. Gespräche mit kirchlichen Professionellen, Familienmitgliedern und Personen jenseits der Familie nach Kirchgangshäufigkeit, in \%.

Die Häufigkeit des Gesprächs mit kirchlichen Professionellen steigt mit der Kirchgangshäufigkeit monoton von $10 \%$ auf 78 \%, um 68 Prozentpunkte an; Eta beträgt .417. Die Häufigkeit des Gesprächs mit Familienmitgliedern steigt von $60 \%$ auf 88 \%, um 28 Prozentpunkte an; Eta beträgt .212. Die Zuständigkeitshypothese und die positive Seite der Bedarfshypothese werden bestätigt. Aber die Wirkung der Kirchgangshäufigkeit auf das Gespräch mit kirchlichen Professionellen ist - nach Prozentsatzdifferenzen wie Eta - doppelt so stark wie auf das Gespräch mit Familienmitgliedern. Denn die Kirchenbindung steigert die Bereitschaft mit kirchlichen Professionellen zu sprechen direkt, die Bereitschaft mit Familienmitgliedern zu sprechen nur indirekt über den gemeinsamen Bedarf.

Die Häufigkeit des Gesprächs mit Personen jenseits der Familie geht zwischen Kirchgang Nie und den drei folgenden Ausprägungen von $78 \%$ auf rund $70 \%$ zurück, um dann bis zur höchsten Ausprägung auf 88\% anzuwachsen. Zwischen den der Kirche am fernsten und am nächsten Stehenden steigt sie zwar um 10 Prozentpunkte; aber die Schwankungen in beide Richtungen sind mit 19 Prozentpunkten fast doppelt so groß. Eta beträgt .107. Auf der einen Seite verlagern die Nie-Kirchgänger offenbar ihren Bedarf an Gesprächen über Religion von den kirchlichen Professionellen auf Personen jenseits der Familie, vielleicht auf säkular an Religion interessierte Personen. Auf der anderen Seite gewinnen die, die wenigstens wöchentlich in die Kirche gehen, offenbar in der Gemeinde außerfamiliäre Kontakte oder gehen aufgrund gemeindlicher Kontakte so häufig in die Kirche. ${ }^{4}$ Aber die beiden extremen Gruppen der Nie-Kirchgänger umfassen nur 24,5\% und der wöchentlichen Kirch-

4 Der Anstieg kann sich nicht aus der verstärkten Nennung der Vorgabe $\mathrm{H}$ „Menschen in kirchlichen oder anderen religiösen Gruppen" ergeben, die zu den kirchlichen Professionellen gerechnet wurden. Er muss sich aus den Vorgaben E „Freunde“ und F „Bekannte, Nachbarn oder Arbeitskollegen“ rekrutieren, mit denen die häufigen Kirchgänger auch religiöse Kontakte pflegen. Um die Gruppe derer zu identifizieren, wo die kirchliche Beziehung die außerfamiliale einschließt, wäre eine Vorgabe "Mitglieder meiner Pfarrgemeinde" erforderlich gewesen. 
gänger nur 9,9 \% derer, die mit anderen über Religion reden, während die Mittelgruppe 65,6 \% umfasst. Die negative Seite der Bedarfshypothese wird also im Schwerpunkt der Befragten bestätigt und in den Extremen durch nachträglich erklärbare Tendenzen überlagert.

Mit steigender Häufigkeit des Kirchgangs spricht man mal mit Familienangehörigen, mal mit Personen jenseits der Familie am häufigsten über Religion. Per saldo werden beide Partner gleich häufig gewählt. Und in allen Kirchgangs-Gruppen liegen die kirchlichen Professionellen nicht vor, sondern weit hinter den Personen jenseits der Familie - um fast 70 Prozentpunkte bei den Nie-Kirchgängern, aber auch bei den eifrigsten Kirchgängern noch um 10 Prozentpunkte. Vielleicht gilt auch hier, dass mit dem häufigen Kirchgang viele Gesprächspartner außerhalb der Familie, aber innerhalb der Gemeinde gefunden werden. Auch bei einer Aufgliederung nach dem Kirchgang wird die Partikularismushypothese nicht bestätigt. Vergleicht man Abbildung 2 mit Abbildung 1, so bilden partikularistische Beziehungen das Dach nicht allein, sondern zusammen mit universalistischen Beziehungen. Und die universalistische Beziehung zu kirchlichen Professionellen gewinnt zwar daraus, dass sie partikularistische Elemente aufnehmen kann, bleibt aber selbst dort, wo die Chance des Gewinns am größten ist, hinter partikularistischen und universalistischen Beziehungen zurück.

Das christliche Weltbild hängt nicht nur in der gesamten Stichprobe mit der Häufigkeit der Diskussion über Religion zusammen ( $r=.38)$, sondern auch in der Gruppe derer, die überhaupt über Religion sprechen, mit der Zahl der genannten Gesprächspartner $(r=.31)$. Für die grafische Darstellung wurde es in fünf ordinale Klassen mit annähernd gleicher Besetzung eingeteilt. Wie sich die Gesprächspartner aus kirchlichen Professionellen, Familienangehörigen und Personen jenseits der Familie in Abhängigkeit von diesen fünf Klassen verteilen, stellt Abbildung 3 dar.

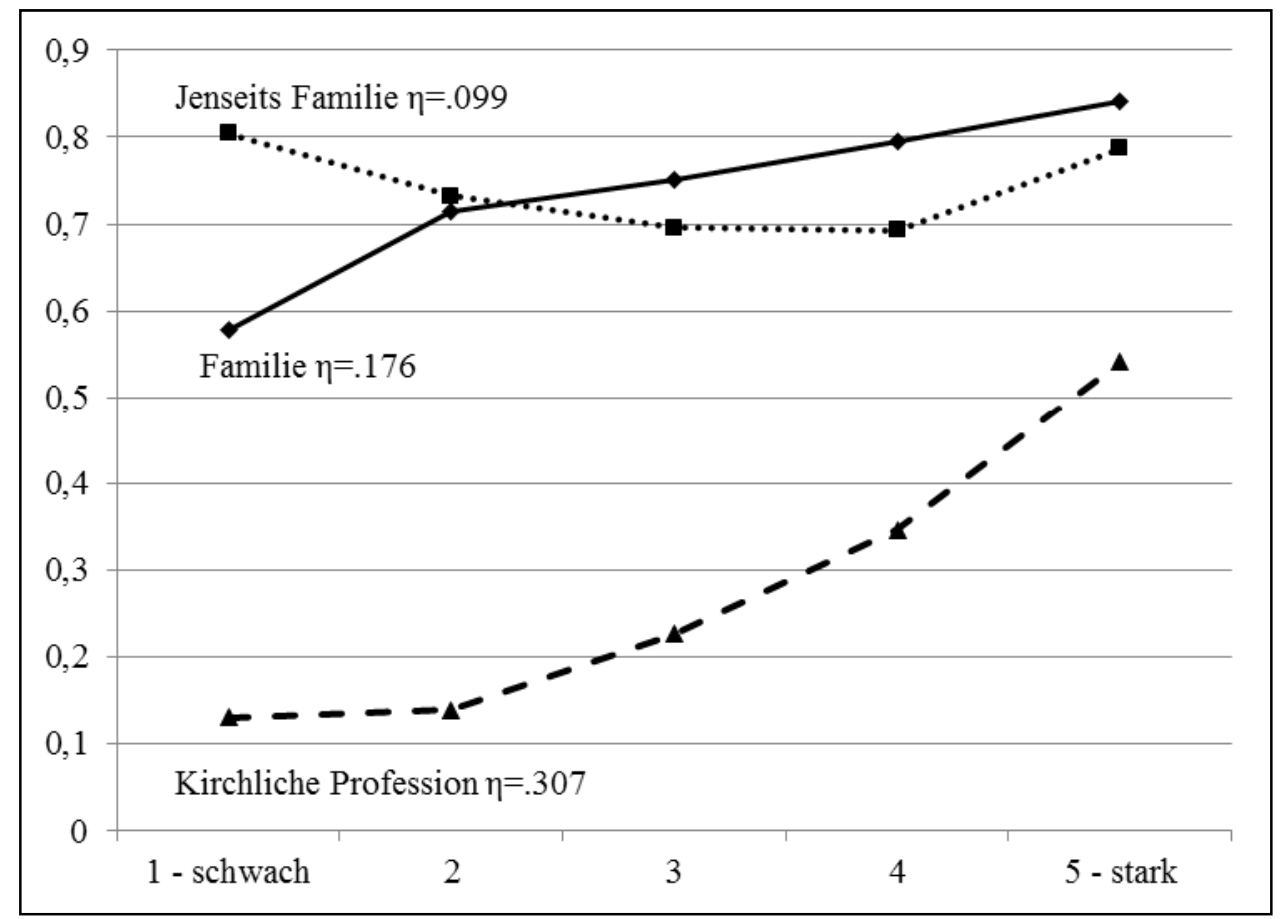

Abbildung 3. Gespräche mit kirchlichen Professionellen, Familienmitgliedern und Personen jenseits der Familie nach der Stärke des christlichen Weltbilds, in \%. 
Die Häufigkeit des Gesprächs mit kirchlichen Professionellen steigt mit dem christlichen Weltbild monoton von $13 \%$ auf 54 \%, um 41 Prozentpunkte an; Eta beträgt .307. Die Häufigkeit des Gesprächs mit Familienmitgliedern steigt von $58 \%$ auf 84 $\%$, um 26 Prozentpunkte an; Eta beträgt .176. Wie beim Kirchgang gilt also: Die Zuständigkeitshypothese und die positive Seite der Bedarfshypothese werden bestätigt und die Wirkung auf das Gespräch mit kirchlichen Professionellen ist deutlich stärker als auf das Gespräch mit Familienmitgliedern.

Die Häufigkeit des Gesprächs mit Personen jenseits der Familie geht zwischen der ersten und dritten sowie vierten Klasse von $80 \%$ auf $70 \%$ zurück, um dann bis zur fünften Klasse auf $79 \%$ anzuwachsen. Über alle Stufen der Kirchenbindung bleibt sie also konstant. Eta beträgt .099. Wie beim Kirchgang gilt: Kirchenferne verlagern ihren Bedarf an Gesprächen über Religion von den religiös Professionellen auf Personen jenseits der Familie; Kirchennahe gewinnen - aufgrund ihres Glaubens - in der Gemeinde außerfamiliale Kontakte. Anders als beim Kirchgang aber gibt es keinen Unterschied zwischen den Extremen und die Differenzen sind schwächer, so dass man die negative Seite der Bedarfshypothese als bestätigt werten kann.

Mit zunehmender Stärke des christlichen Weltbildes spricht man mal mit Familienangehörigen, mal mit Personen jenseits der Familie am häufigsten über Religion. Per saldo werden beide Partner gleich häufig gewählt. Und unabhängig von der Stärke des christlichen Weltbildes liegen die kirchlichen Professionellen nicht vor, sondern weit hinter den Personen jenseits der Familie - um fast 67 Prozentpunkte in der untersten, aber auch in der obersten Gruppe noch um 25 Prozentpunkte. Wiederum wird die Partikularismushypothese nicht bestätigt: Partikularistische und universalistische Beziehungen werden am häufigsten gewählt, und die kirchlichen Professionellen sind weit abgeschlagen.

Das naturalistische Weltbild hängt nicht nur in der gesamten Stichprobe negativ mit der Häufigkeit der Diskussion über Religion zusammen ( $r=-.22)$, sondern auch in der Gruppe derer, die überhaupt über Religion sprechen, mit der Zahl der genannten Gesprächspartner $(r=-.29)$. Wie das christliche wurde das naturalistische Weltbild für die grafische Darstellung in fünf ordinale Klassen mit annähernd gleicher Besetzung eingeteilt. Wie sich die Gesprächspartner aus kirchlichen Professionellen, Familienangehörigen und Personen jenseits der Familie in Abhängigkeit von diesen fünf Klassen verteilen, stellt Abbildung 4 dar. Weil die Stärke des naturalistischen Weltbildes Kirchenferne indiziert, wurde die Anordnung der ordinalen Klassen umgekehrt. 


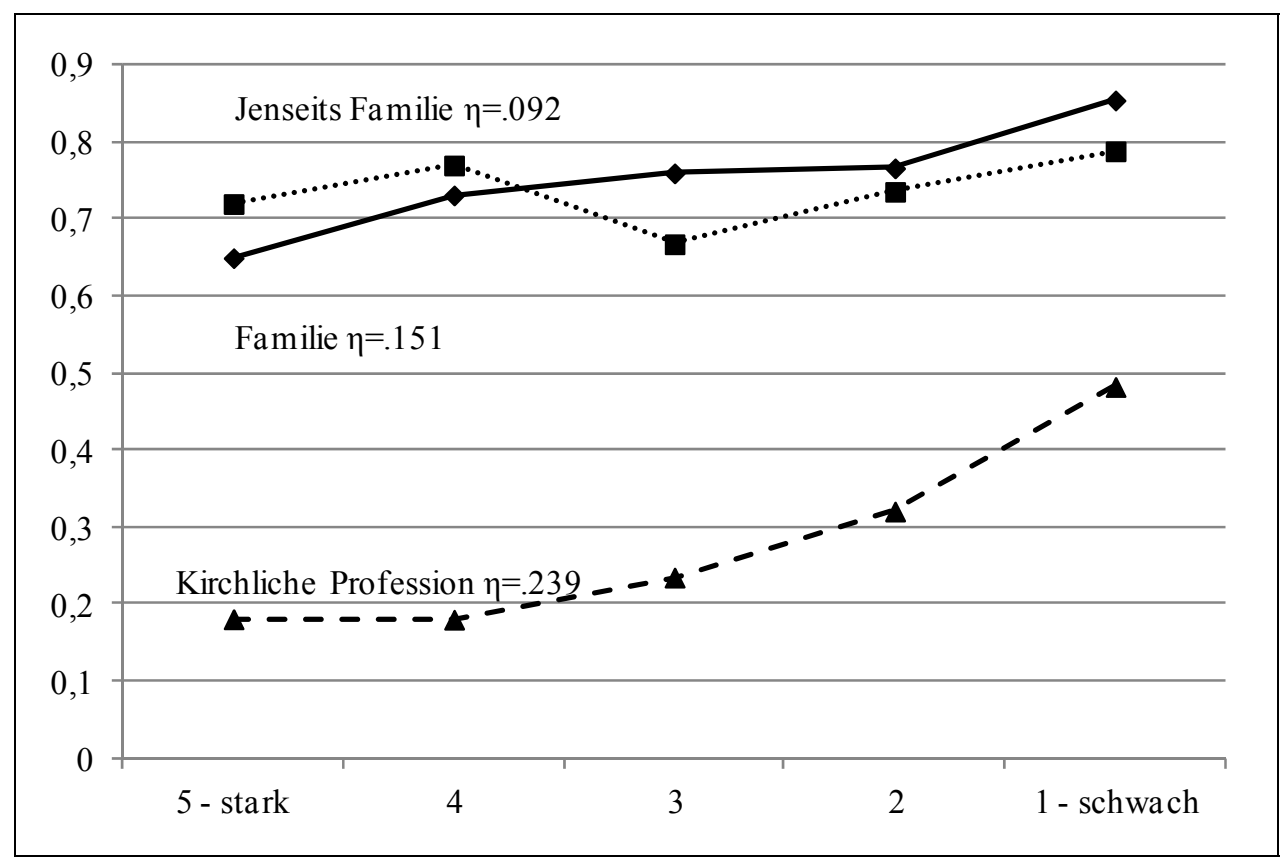

Abbildung 4. Gespräche mit kirchlichen Professionellen, Familienmitgliedern und Personen jenseits der Familie nach der Stärke des naturalistischen Weltbildes, in \%.

Mit dem Rückgang des naturalistischen Weltbilds steigt die Häufigkeit des Gesprächs mit kirchlichen Professionellen monoton von $18 \%$ auf $48 \%$, um 30 Prozentpunkte an; Eta beträgt .239. Und die Häufigkeit des Gesprächs mit Familienmitgliedern steigt von $65 \%$ auf $85 \%$, um 20 Prozentpunkte an; Eta beträgt .151. Die Zuständigkeitshypothese und die positive Seite der Bedarfshypothese werden bestätigt. Aber die negative Wirkung des naturalistischen Weltbilds auf das Gespräch mit kirchlichen Professionellen ist stärker als auf das Gespräch mit Familienmitgliedern. Die Häufigkeit des Gesprächs mit Personen jenseits der Familie steigt zwar vom stärksten bis zum schwächsten naturalistischen Weltbild von $72 \%$ auf $79 \%$, um 7 Prozentpunkte an, schwankt aber unregelmäßig; Eta beträgt .092. Insgesamt hat das naturalistische Weltbild keinen Einfluss; die negative Seite der Bedarfshypothese wird bestätigt.

Mit abnehmender Stärke des naturalistischen Weltbildes spricht man mal mit Familienangehörigen, mal mit Personen jenseits der Familie am häufigsten über Religion. Und unabhängig von der Schwäche des naturalistischen Weltbildes liegen die kirchlichen Professionellen nicht vor, sondern weit hinter den Personen jenseits der Familie - um 64 Prozentpunkte in der stärksten und in der schwächsten Gruppe noch um 30 Prozentpunkte. Wiederum wird die Partikularismushypothese nicht bestätigt: Partikularismus und Universalismus spannen nicht die Breite der Häufigkeiten auf, und die von Universalismus zu Partikularismus changierende Beziehung zu kirchlichen Professionellen liegt nicht dazwischen.

Die Wichtigkeit von Religion und Kirche hängt nicht nur in der gesamten Stichprobe mit der Häufigkeit der Diskussion über Religion zusammen ( $r=.38)$, sondern auch in der Gruppe derer, die überhaupt über Religion sprechen, mit der Zahl der genannten Gesprächspartner ( $r=.31)$. 


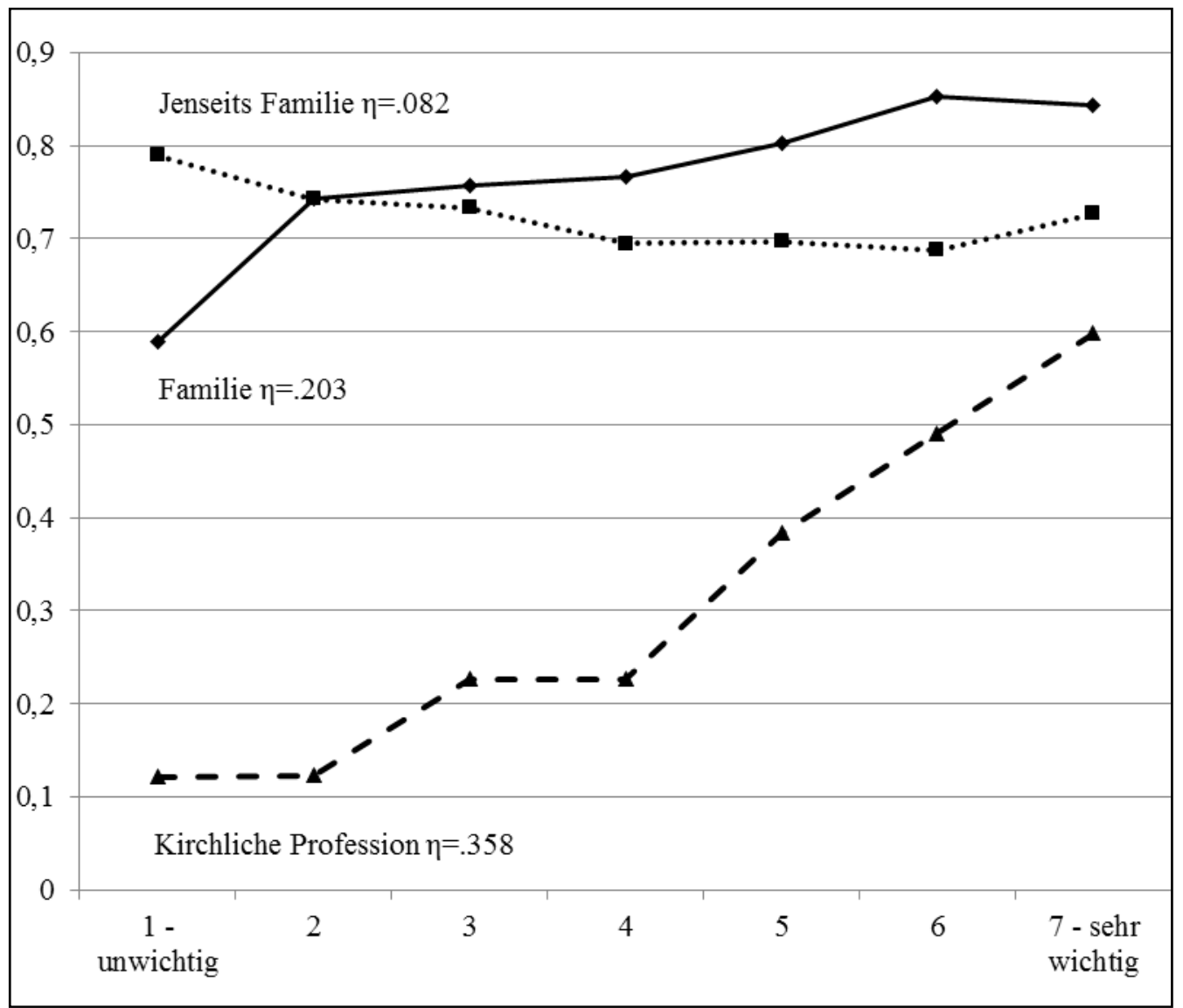

Abbildung 5. Gespräche mit kirchlichen Professionellen, Familienmitgliedern und Personen jenseits der Familie nach der Wichtigkeit von Religion und Kirche, in \%.

Mit der Wichtigkeit von Religion und Kirche steigt die Häufigkeit des Gesprächs mit kirchlichen Professionellen monoton von 12 \% auf 49 \%, um 37 Prozentpunkte an; Eta beträgt .358. Und die Häufigkeit des Gesprächs mit Familienmitgliedern steigt von 59 \% auf 84 \%, um 25 Prozentpunkte an; Eta beträgt .203. Die Zuständigkeitshypothese und die positive Seite der Bedarfshypothese werden bestätigt. Aber die Wirkung auf das Gespräch mit kirchlichen Professionellen ist stärker als auf das Gespräch mit Familienmitgliedern. Die Häufigkeit des Gesprächs mit Personen jenseits der Familie sinkt von der untersten bis zur sechsten Stufe der Wichtigkeit von $79 \%$ auf $69 \%$, um 10 Prozentpunkte und steigt auf der höchsten Stufe wieder auf $63 \%$ an; Eta beträgt .082. Insgesamt hat die Wichtigkeit von Religion und Kirche keinen Einfluss; die negative Seite der Bedarfshypothese wird bestätigt.

Mit der Wichtigkeit von Religion und Kirche spricht man mal mit Familienangehörigen, mal mit Personen jenseits der Familie am häufigsten über Religion. Und unabhängig von der Wichtigkeit liegen die kirchlichen Professionellen nicht vor, sondern weit hinter den Personen jenseits der Familie - um 67 Prozentpunkte in der untersten und in der obersten Gruppe noch um 20 Prozentpunkte. Wiederum wird die Partikularismushypothese nicht bestätigt.

Die Variablen zu Kirchenbindung - Kirchgang, christliches und naturalistisches Weltbild, Wichtigkeit von Religion und Kirche - beeinflussen die Wahl der Partner für ein Gespräch über Religion nach dem gleichen Muster. Die Partikularismushypothese wird nicht, die Zuständigkeitshypothese und die positive Seite der Bedarfshypothese 
werden voll, die negative Seite der Bedarfshypothese wird näherungsweise bestätigt. Aber die Stärke der Einflüsse unterscheidet sich. Gemessen an Prozentsatzdifferenzen und Eta-Werten hängt die Wahl der Partner am stärksten vom Kirchgang ab, gefolgt von der Wichtigkeit von Religion und Kirche, vom christlichen und schließlich dem naturalistischen Weltbild. Der stärkste und zweitstärkste Zusammenhang besteht zwischen dem Kirchgang und dem Gespräch mit kirchlichen Professionellen sowie der Wichtigkeit von Religion und Kirche. Die kirchliche Praxis ist in höherem Maße Basis für das Gespräch über Religion als religiöse Überzeugungen. Das Gespräch über Religion gleich mit wem wird durch den sozialen Kontakt mit der Kirche stärker als durch die persönliche Auseinandersetzung über Glaubensfragen gefördert.

\subsection{Opportunitäten und Kontrollvariablen}

Die Korrelationen des privaten Lebenslaufs mit der Wahl eines Partners für ein Gespräch über Religion sind in Tabelle 1 dargestellt. Die Erwartung, dass Wendepunkte des privaten Lebens zum Gespräch mit kirchlichen Professionellen bewegen, gilt nicht für die Ehe, wohl aber - in schwachem Maße - die Elternschaft. Ehe wie Elternschaft sind als Stufen der Familienbildung aber wie erwartet auch Bedingung der Wahl von Familienmitgliedern. Die Wahl von Personen jenseits der Familie schließlich ist entgegen der Erwartung nicht unabhängig von Ehe und Elternschaft, sondern negativ mit innen korreliert.

Das Muster der Lebenslaufhypothese erscheint also nur partiell: Übergänge des privaten Lebenslaufs bringen die Familie nur ein wenig näher zur Kirche, aber enger zusammen; und sie rücken das Umfeld der Familie in den Hintergrund. Die Kirchen, die den Auftrag haben, die im Lebenszyklus variierenden Chancen der religiösen Kommunikation zu nutzen, gewinnen nicht; die Umwelt der Familie, die keinen religiösen Auftrag hat, verliert. Dass die Kirchen ihre Chancen nicht steigern können, ist wohl weniger Versagen ihrer Anbieter als Spiegel der vorherrschenden Nachfrage an Wendepunkten des privaten Lebens. Sie richtet sich auf die zeremonielle Begleitung der Anlässe und geht wenig darüber hinaus. Dass nach privaten Übergängen die Gespräche über Religion mit Personen jenseits der Familie zurückgehen, ist vermutlich Teil eines allgemeinen Rückzugs aus der Öffentlichkeit im Familienzyklus: Die Partner konzentrieren sich aufeinander und die Kinder, so dass Kontakte nach außen überhaupt schwächer werden - und mit ihnen auch Gespräche über Religion. 


\begin{tabular}{|lllll|}
\hline & $\mathrm{H}$ & KIRPROF & FAMILIE & JENFAM \\
EHE & ++0 & $(.03)$ & .24 & -.17 \\
KIND & ++0 & .07 & .16 & -.14 \\
ALTER - & & $(.04)$ & $(.02)$ & -.11 \\
ALTERQU & & $(-.01)$ & -.12 & $(.03)$ \\
\hline
\end{tabular}

$\mathrm{N}$ mindestens 2741 , alle Koeffizienten signifikant $\mathrm{p}<.001$, außer denen in Klammern. $\mathrm{H}$ : Hypothesenmuster.

Tabelle 1 Korrelationen des privaten Lebenslaufs mit der Wahl von Partnern für ein Gespräch über Religion

Mit dem linearen Alter gehen Gespräche über Religion mit Personen jenseits der Familie zurück. Auch dahinter könnte der allgemeine Rückzug aus der Öffentlichkeit im Familienzyklus stehen. Da aber das lineare Alter stark mit Heirat und Elternschaft korreliert ( $r=.32$ und .46), kann diese Erklärung erst in der multivariaten Analyse geprüft werden. Das quadrierte Alter korreliert negativ mit der Wahl von Familienangehörigen; das heißt: in der Lebensmitte wird häufiger als in der Jugend und im hohen Alter mit Familienangehörigen über Religion gesprochen. Offenbar ist man in der Lebensmitte herausgefordert, über den Sinn der programmierten Übergänge im Rahmen des ganzen Lebens nachzudenken; aber das Nachdenken mündet in ein Gespräch über Religion nur in, nicht außerhalb der Familie - insbesondere nicht in ein Gespräch mit kirchlichen Professionellen.

Die Korrelationen von Ressourcen und Kontrollvariablen mit der Wahl eines Partners für ein Gespräch über Religion sind in Tabelle 2 dargestellt. Vertrauen in Fremde ist wie erwartet für ein Gespräch mit Familienmitgliedern nicht, wohl aber für ein Gespräch mit Personen jenseits der Familie und auch - entgegen der Erwartung kirchlichen Professionellen erforderlich. Offenbar sieht, wer überhaupt über Religion spricht, die Beziehung zu kirchlichen Professionellen eher universalistisch als partikularistisch. ${ }^{5}$ Weiterhin korreliert wie erwartet der Sozialstatus - Bildung und Berufsprestige - nicht mit der Wahl von kirchlichen Professionellen und Familienmitgliedern, aber deutlich mit Personen jenseits der Familie. Die Ressourcenhypothese wird also für Sozialstatus, nicht aber durchgehend für Vertrauen bestätigt: Für ein Gespräch über Religion mit Familienmitgliedern braucht man kein Vertrauen in Fremde, wohl aber für ein Gespräch mit Personen jenseits der Familie und auch mit kirchlichen Professionellen.

5 Man kann vermuten, dass die Kirchenbindung, die die Beziehung zu kirchlichen Professionellen näher zum Partikularismus rückt, den Zusammenhang moderiert. Unter Kirchennahen wäre dann Vertrauen keine Voraussetzung für ein Gespräch mit kirchlichen Professionellen, unter Kirchenfernen aber umso mehr. 


\begin{tabular}{|lllll|}
\hline & $\mathrm{H}$ & KIRPROF & FAMILIE & JENFAM \\
VERTRAUEN & $00+$ & .08 & $(.05)$ & .09 \\
BILDUNG & $00+$ & $(.05)$ & $(.06)$ & .14 \\
BP-ISEI_ & $00+$ & $(.05)$ & $(.04)$ & .13 \\
EVAN & & $(.05)$ & $(-.00)$ & $(-.03)$ \\
KATH & & .09 & .13 & $(-.04)$ \\
FRAU & & .06 & $(-.01)$ & $(.04)$ \\
OST & & $(-.01)$ & -.10 & $(.03)$ \\
\hline
\end{tabular}

$\mathrm{N}$ mindestens 2486, alle Koeffizienten signifikant $\mathrm{p}<.001$, außer denen in Klammern. $\mathrm{H}$ : Hypothesenmuster

Tabelle 2 Korrelationen von Ressourcen und Kontrollvariablen mit der Wahl von Partnern für ein Gespräch über Religion

Mitglieder der evangelischen Kirche, die überhaupt über Religion sprechen, wählen ihre Gesprächspartner nicht signifikant anders als Konfessionslose - so wenig wie in der Gesamtstichprobe alle Mitglieder der evangelischen Kirche häufiger als Konfessionslose überhaupt über Religion sprechen (Meulemann, 2014a). Insbesondere sprechen Evangelische nicht häufiger mit kirchlichen Professionellen als Konfessionslose - in Kontrast zu dem Ergebnis 2002, dass Evangelische weitaus häufiger als Konfessionslose mit Pfarrern über Religion sprechen (Huber et al., 2006, S. 462). Mitglieder der katholischen Kirche, die überhaupt über Religion sprechen, sprechen zwar signifikant häufiger mit kirchlichen Professionellen als Konfessionslose - so wie in der Gesamtstichprobe alle Mitglieder der katholischen Kirche häufiger als Konfessionslose überhaupt über Religion sprechen (Huber et al., 2006, S. 462). Die Korrelationen sind zwar schwach. Aber sie widersprechen der Erwartung, dass unter Protestanten das größere dogmatische und praktische Gewicht der Gemeinde sich in einen engeren Kontakt zwischen Pastor und Gläubigen niederschlägt. Vielleicht wirkt sich Luthers Lehre vom Priesterstand aller Gläubigen heute noch so aus, dass Protestanten weniger Bedarf an Gesprächen mit Seelsorgern haben als Konfessionslose und als Katholiken. Schließlich sprechen Katholiken häufiger mit Familienmitgliedern als Konfessionslose und Protestanten.

Der schwache Einfluss der Konfessionsmitgliedschaft auf die Wahl der Gesprächspartner und insbesondere die Wahl kirchlicher Professioneller kontrastiert scharf zu dem starken Einfluss der Kirchenbindung. Die Mitgliedschaft ist nicht deckungsgleich mit sozialer Teilhabe in der Gemeinde; sie ebnet anders als die Kirchenbindung nicht den Weg ins Pfarr- oder Gemeindehaus.

Frauen sprechen eher mit kirchlichen Professionellen als Männer, Ostdeutsche weniger mit Familienmitgliedern als Westdeutsche. Offenbar hat der Kampf der DDR gegen die Kirchen bis in die Familien gewirkt.

\section{Ergebnisse multivariat}

Die logistischen Regressionen der Gespräche mit kirchlichen Professionellen, Familienmitgliedern und Personen jenseits der Familie auf Kirchenbindung, Opportunitäten und Kontrollvariablen sind in Tabelle 3 wiedergegeben.

\begin{tabular}{|lll|}
\hline KIRPROF & FAMILIE & JENFAM \\
\hline
\end{tabular}




\begin{tabular}{|c|c|c|c|c|c|c|c|}
\hline & $\mathrm{H}$ & Roh & stand & Roh & stand & Roh & stand \\
\hline Konstante & & -3.322 & & .494 & & .612 & \\
\hline KIRCHG & ++0 & $1.741^{\star \star \star}$ & 2.061 & 1.125 & 1.278 & $1.193^{\star \star}$ & 1.258 \\
\hline CHRI & ++0 & $1.158^{\star}$ & 1.188 & 1.113 & 1.134 & 1.061 & 1.072 \\
\hline NAT & --0 & $.857^{\star \star *}$ & $1.184^{-1}$ & $.877^{*}$ & $1.154^{-1}$ & .957 & $1.049^{-1}$ \\
\hline WRELIFAM & ++0 & $1.172^{\star \star \star}$ & 1.363 & $1.124^{\star *}$ & 1.257 & .931 & $1.149^{-1}$ \\
\hline EHE & ++0 & $.740^{\star}$ & $1.158^{-1}$ & $2.776^{\star \star \star}$ & 1.644 & 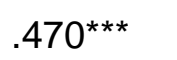 & $1.444^{-1}$ \\
\hline KIND & ++0 & $1.443^{*}$ & 1.444 & 1.590 *** & 1.235 & .773 & $1.125^{-1}$ \\
\hline ALTER & & $.990^{\star}$ & $1.179^{-1}$ & $.982^{\star \star \star}$ & $1.344^{-1}$ & 1.002 & 1.033 \\
\hline ALTERQU & & 1.000 & 1.000 & 1.000 & 1.000 & 1.000 & 1.000 \\
\hline VERTRAU & $00+$ & $1.201^{\star *}$ & 1.148 & 1.041 & 1.031 & $1.144^{*}$ & 1.107 \\
\hline BILDUNG & $00+$ & 1.069 & 1.107 & $1.130^{*}$ & 1.204 & $1.110^{*}$ & 1.170 \\
\hline BP-ISEI & $00+$ & 1.007 & 1.105 & .999 & $1.016^{-1}$ & $1.011^{\star}$ & 1.187 \\
\hline EVAN & & .964 & $1.019^{-1}$ & .924 & 1.039 & $.654^{\star *}$ & $1.228^{-1}$ \\
\hline KATH & & .759 & $1.143^{-1}$ & 1.126 & 1.060 & $.599 * \star$ & $1.284^{-1}$ \\
\hline FRAU & & 1.038 & 1.019 & .885 & $1.063^{-1}$ & $1.233^{*}$ & 1.111 \\
\hline OST & & $1.517^{\star \star \star}$ & 1.163 & $.607^{\star \star \star}$ & $1.197^{-1}$ & 1.065 & 1.024 \\
\hline $\mathrm{R}^{2}$ (alle, 16) & & .273 & & .171 & & .095 & \\
\hline $\mathrm{R}^{2}(\mathrm{~KB}, 5)$ & & $.247(90)$ & & $.076(44)$ & & $.019(20)$ & \\
\hline $\mathrm{R}^{2}(\mathrm{O}+\mathrm{K}, 12)$ & & $.079(29)$ & & $.137(80)$ & & $.085(90)$ & \\
\hline
\end{tabular}

$\mathrm{H}$ : Hypothesenmuster. Stand = $\mathrm{x}$-standardisiert. $\mathrm{R}^{2}$ Nagelkerke, Prädiktorgruppe und Freiheitsgrade in Klammer. N(gewichtet mit V743 für Gesamtdeutschland): 2451 für alle, 2739 für Kirchenbindung (KB), 2461 für Opportunitäten+Kontrolle $(\mathrm{O}+\mathrm{K})$ bei jeder Zielvariablen. In Klammer nach $\mathrm{R}^{2}$-Werten: Anteil am $\mathrm{R}^{2}$-Wert für alle. ${ }^{* \star *} \mathrm{p}<.001,{ }^{* \star} \mathrm{p}<.01,{ }^{\star} \mathrm{p}<.05$.

Tabelle 3 Logistische Regressionen des Gesprächs mit kirchlichen Professionellen, Familienmitgliedern und Personen jenseits der Familie auf Kirchenbindung, Opportunitäten und Kontrollvariablen, Chancenverhältnisse

\section{Einfluss der Prädiktorgruppen auf die Wahlen insgesamt}

Die Kirchenbindung bestimmt die Wahl von kirchlichen Professionellen und Familienmitgliedern über die positiven Indikatoren des Kirchgangs, des christlichen Weltbilds und der Wichtigkeit von Religion und Kirche - meist signifikant - positiv und über den negativen Indikator des naturalistischen Weltbilds signifikant negativ. Die Zuständigkeitshypothese und die positive Seite der Bedarfshypothese werden bestätigt. Die Wahl von Personen jenseits der Familie aber bestimmt die Kirchenbindung nur noch als Kirchgang. Die negative Seite der Bedarfshypothese wird also zumindest tendenziell bestätigt. Gemessen an den standardisierten Koeffizienten dieser Prädiktorgruppe ist der Einfluss des Kirchengangs auf alle drei Wahlen der stärkste, gefolgt von der Wichtigkeit von Religion und Kirche. Auch in multivariater Betrachtung ist die kirchliche Praxis folgenreicher für die Wahl eines Gesprächspartners als der Glaube. 
Mit Blick auf den privaten Lebenslauf hängen Gespräche mit kirchlichen Professionellen negativ von der Ehe und positiv von der Elternschaft ab; Gespräche mit Familienangehörigen und mit Personen jenseits der Familie hängen positiv bzw. negativ von Ehe wie Elternschaft ab. Das Muster der Lebenslaufhypothese wird also noch weniger sichtbar als bei der bivariaten Betrachtung. ${ }^{6}$ Aber auch jetzt bleibt der Gegensatz zwischen der Wahl von kirchlichen Professionellen und Familienmitgliedern einerseits und von Personen jenseits der Familie anderseits erhalten. Das lineare Alter hat einen negativen Einfluss auf Gespräche mit kirchlichen Professionellen und Familienangehörigen; das quadrierte Alter hat keinen Einfluss. Gemessen an den standardisierten Koeffizienten dieser Prädiktorgruppe hängen die Wahl kirchlicher Professioneller am stärksten von der Elternschaft, und die Wahl von Familienmitgliedern und Personen jenseits der Familie am stärksten von der Ehe ab.

Das Vertrauen hat auf das Gespräch mit kirchlichen Professionellen und Personen jenseits der Familie einen positiven, auf das Gespräch mit Familienmitgliedern aber keinen Einfluss. Wie die bivariate legt es also auch die multivariate Analyse nahe, die Beziehung zu kirchlichen Professionellen eher universalistisch als partikularistisch zu sehen. Die Bildung beeinflusst wie erwartet Gespräche mit kirchlichen Professionellen nicht und Gespräche mit Personen jenseits der Familie positiv, aber wider Erwarten auch Gespräche mit Familienmitgliedern positiv; während der Berufsstatus mit allen drei Gesprächspartnern wie erwartet zusammenhängt. Die Ergebnisse sind mit Muster der Ressourcenhypothese bis auf zwei Diskrepanzen vereinbar.

Die Konfessionsmitgliedschaft hat auf Gespräche mit kirchlichen Professionellen keinen signifikanten Einfluss. Während Katholiken, wenn der Kirchgang nicht kontrolliert ist, häufiger mit kirchlichen Professionellen als Protestanten und Konfessionslose sprechen (siehe Tabelle 2), dreht sich bei Kontrolle des Kirchgangs die Beziehung um: Katholiken sprechen - allerdings nicht signifikant - seltener mit kirchlichen Professionellen als Protestanten und Konfessionslose. Das könnte eine Folge der „Privilegierung ritueller Kommunikation“ (Breuer, 2012, S.422-436, 441-444) sein. Weil im Katholizismus die religiöse Kommunikation im Vollzug der Liturgie gegenüber der Kommunikation in der Seelsorge „privilegiert“ wird, sprechen Katholiken, die durch den Kirchgang bereits ihren Bedarf an religiöser Kommunikation befriedigt haben, seltener mit ihrem Pfarrer als Protestanten. Wie dem auch sei, die schwachen und nicht signifikanten Einflüsse der Konfessionsmitgliedschaft auf das Gespräch mit kirchlichen Professionellen kontrastieren mit dem starken Einfluss der Kirchenbindung. Wie erwartet ist nicht die Mitgliedschaft, sondern die Teilhabe Basis des Gesprächs mit kirchlichen Professionellen.

Die Konfessionsmitgliedschaft hat allerdings auf Gespräche mit Personen jenseits der Familie einen negativen Einfluss. Konfessionslose sprechen also häufiger als Katholiken und Protestanten mit Personen jenseits der Familie über Religion - vielleicht weil kirchliche Professionelle für sie keine Alternative sind.

Frauen sprechen mit Personen jenseits der Familie häufiger als Männer. Ostdeutsche sprechen mit kirchlichen Professionellen häufiger als Westdeutsche; wenn die geringere Kirchenbindung der Ostdeutschen kontrolliert ist, wenden sie sich häufiger

6 Die hohe Korrelation zwischen Ehe und Elternschaft $(r=.52)$ und die Korrelationen beider mit dem linearen und quadrierten Alter ( $r=.32$ und $r=.46$ bzw. $r=-.30$ und $r=-.26)$ - Ehe und Elternschaft nehmen mit dem Alter zu und finden sich besonders häufig im mittleren Alter - sind kontrolliert. Weil bereits die bivariaten Korrelationen von Ehe und Elternschaft mit der Wahl kirchlicher Professioneller geringer waren als mit der Wahl von Familienmitgliedern und Personen jenseits der Familie, wird der Regressionskoeffizient für die Ehe negativ. 
als Westdeutsche an kirchliche Professionelle. Ostdeutsche sprechen weiterhin seltener mit Familienmitgliedern als Westdeutsche.

Die Opportunitäten und Kontrollvariablen insgesamt wirken in drei Weisen entgegengesetzt auf die Wahl zwischen Personen in und außerhalb der Familie. Erstens ist der Status des privaten Lebenslaufs Bedingung für Gespräche mit Familienangehörigen, und hält von Gesprächen mit Personen jenseits der Familie ab. Zweitens sind Sozialstatus und Vertrauen für Gespräche mit Familienangehörigen überwiegend belanglos, und für Gespräche mit Personen jenseits der Familie förderlich. Drittens ist die Konfessionsmitgliedschaft für Gespräche mit Familienangehörigen unerheblich, und für Gespräche mit Personen jenseits der Familie demotivierend. In allen drei Sichtweisen stehen sich Gespräche mit Familienangehörigen Gesprächen mit Personen jenseits der Familie gegenüber. In religiösen Fragen tut man sich mit Familienangehörigen zusammen, aber wendet sich kaum nach außen. Die Grenze der Gesprächssuche liegt zwischen partikularistischen und universalistischen Beziehungen.

\section{Vergleich der Einflussstärken zwischen den Wahlen}

Vergleicht man zwischen den Wahlen die Rangfolge der standardisierten Koeffizienten aller Prädiktorgruppen, so wird die Wahl kirchlicher Professioneller am stärksten durch den Kirchgang, die Wahl von Familienmitgliedern und von Personen jenseits der Familie am stärksten durch die Ehe beeinflusst. Kirchliche Professionelle werden mit der Kirchenbindung stärker gewählt; Familienmitglieder und Personen jenseits der Familie nach den Opportunitäten des fortschreitenden Familienzyklus - die ersten mehr, die zweiten weniger. Die dominante Orientierungspunkte unterscheiden sich: Die Wahl kirchlichen Professionellen folgt der Kirchenbindung, die Wahl von Personen in und jenseits der Familie positiv oder negativ dem Familienzyklus.

Der stärkste standardisierte Koeffizient erfasst aber nur gleichsam die Speerspitze, nicht aber die gesamte Orientierungskraft von Kirchenbindung und Opportunitäten. Sie lässt sich am Anteil der $\mathrm{R}^{2}$-Werte für die Kirchenbindung einerseits, die Opportunitäten und Kontrollvariablen anderseits an den $\mathrm{R}^{2}$-Werten für alle Prädiktorgruppen als Prozentsatz (in Klammern) ermessen.

Die Kirchenbindung trägt zur Gesamterklärung der Gespräche mit kirchlichen Professionellen sehr viel, der Gespräche mit Familienmitgliedern etwas und der Gespräche mit Personen jenseits der Familie sehr wenig bei. Im Paket der Prädiktorgruppen gilt: Zuständigkeits- und Bedarfshypothese, also die differentielle Wirkung der Kirchenbindung auf die Wahl der Partner, werden bestätigt; die Kirchenbindung ist der dominante Orientierungspunkt für die Wahl kirchlicher Professioneller.

Die Opportunitäten und Kontrollvariablen tragen zur Gesamterklärung der Gespräche mit kirchlichen Professionellen etwas, mit Familienmitgliedern viel und Personen jenseits der Familie sehr viel bei. Im Paket der Prädiktorgruppen gilt: Familienbeziehungen sind für die Wahl von Partnern in und außerhalb der Familie am wichtigsten; Opportunitäten sind also der dominante Orientierungspunkt für die Wahl von Gesprächspartnern jenseits kirchlicher Professioneller.

\section{Ausblick: Nachfrage nach Gesprächen über Religion - und das Angebot?}

Spricht, wer überhaupt über Religion spricht, häufiger mit kirchlichen Professionellen, Familienmitgliedern oder Personen jenseits der Familie? Sieht man die Beziehungen zu diesen Partnern in der Dimension Partikularismus-Universalismus (Parsons \& 
Shils, 1951), so sollte man erwarten, dass Gespräche mit Familienmitgliedern häufiger als mit Personen jenseits der Familie geführt werden und die Häufigkeit des Gesprächs mit kirchlichen Professionellen dazwischen liegt. Tatsächlich aber sind Gespräche mit Familienmitgliedern und mit Personen jenseits der Familie gleich häufig und häufiger als mit kirchlichen Professionellen. Warum wird mit kirchlichen Professionellen so selten und mit Personen jenseits der Familie so häufig über Religion gesprochen? Eine nahe liegende Erklärung liegt in Unterschieden der Zugänglichkeit und der Wertschätzung beider Gruppen.

Kirchliche Professionelle kennen nur wenige, und noch weniger haben zu ihnen Kontakt. Unter den Evangelischen in Deutschland 2012 kannten $23 \%$ keine(n) Pfarrer(in), $33 \%$ hatten trotz Kenntnis keinen Kontakt, und nur 54\% hatten einen „Sprechkontakt“ (Hermelink, Liskowsky \& Grubauer, 2014, S. 96). Vermutlich sind die Zahlen bei Katholiken etwas günstiger und bei Nichtmitgliedern deutlich ungünstiger - in der Gesamtbevölkerung daher kaum besser. Nur die knappe Hälfte der Bevölkerung trifft also im Alltag auf kirchliche Professionelle, so dass die Chance eines Gesprächs sich eröffnet. Zudem sind Religion und Kirche, die die kirchlichen Professionellen ja repräsentieren, in der siebenstufigen Wichtigkeitsschätzung der deutschen Bevölkerung im ALLBUS 2012 (eigene Auswertung) der bei weitem unwichtigste Lebensbereich - fast dreieinhalb Skalenpunkte hinter dem mit 6.5 wichtigsten Lebensbereich der Familie. Die Bevölkerung hat also nur wenig Chancen und nur schwache Motive, mit kirchlichen Professionellen zu sprechen. Beides sind Facetten der Säkularisierung, deren Kehrseite sich im vorliegenden Querschnitt als starker Einfluss der Kirchenbindung auf das Gespräch mit kirchlichen Professionellen wiederfindet.

Aber auch die am stärksten kirchlich Gebundenen sprechen mit kirchlichen Professionellen seltener über Religion als mit Familienmitgliedern und Personen jenseits der Familie. Warum sprechen sie selbst mit universalistischen Partnern häufiger als mit kirchlichen Professionellen? Vielleicht ist ihr religiöses Bedürfnis bereits durch die Kirchennähe in besonderem Maße befriedigt, so dass sie Gespräche über Religion einerseits weniger in der Kirche und anderseits mehr mit „der Welt" führen. Während der große Häufigkeitsvorsprung nichtkirchlicher vor kirchlichen Partnern bei den Kirchenfernen mangelnde Kontakte mit der Kirche spiegelt, könnte der immer noch beträchtliche Vorsprung bei den Kirchennahen sich aus einer Sättigung mit Kontakten ergeben, die eher dem Gemeindeleben als der „Religion“ gewidmet sind.

Personen jenseits der Familie zu treffen, steht auf dem Programm des Lebenslaufs. Zudem gelten Beruf, Freizeit und Freunde im ALLBUS (eigene Auswertung) nach der Familie gleichrangig als die nach der Familie zweitwichtigsten Lebensbereiche. Die Chance eines Gesprächs ist daher sehr hoch, aber die häufige Wahl des Themas Religion bleibt erklärungsbedürftig. Vielleicht ist die Langfristigkeit der Beziehungen ein Schlüssel. Arbeitskollegen trifft man täglich, Freunde regelmäßig. Je häufiger man sich trifft, desto eher sind die persönlich nicht verbindlichen Themen vom Sport bis zur Politik durchgearbeitet, desto eher kann man sich persönlichen Themen wie der Religion widmen. Ein weiterer Schlüssel könnte sein, dass Personen jenseits der Familie als Ersatz für kirchliche Professionelle dienen. Unter innen sind sicher auch säkulare Experten für die religiöse Frage - Therapeuten, Berater, Lehrer. Sieht man von der seelsorgerischen Kernaufgabe der Verkündigung ab, so erfüllen sie die gleiche „Funktion“ wie Seelsorger (Ziemann, 2007, S. 265-274); sie leisten für und mit ihren Klienten „Kontingenzarbeit“ zur Bewältigung des Lebens durch „sinnvolle“ Deutungen der Welt (Schützeichel, 2010). Indiz ihrer Austauschbarkeit ist hier, dass nach allen vier Indikatoren besonders Kirchenferne Personen jenseits der Familie noch 
häufiger als Familienmitglieder wählen. Sie meiden die kirchlichen Professionellen und suchen religiös kompetente nichtkirchliche Ansprechpartner.

Wie immer die Erklärung sein mag, die Häufigkeit der Wahl von Personen jenseits der Familie spricht dagegen, dass Religion in der Bevölkerung als ein persönliches, intimes Thema gesehen wird. Wie über Politik und Wirtschaft, Kultur und Sport, so spricht man mit Arbeitskollegen, Freunden und Nachbarn auch über Religion. Man könnte vermuten: Die Säkularisierung lenkt das religiöse Bedürfnis nicht nur von den Kirchen weg, sondern auch in die Öffentlichkeit; sie ist für die Kirchen schmerzhaft, aber sie aktualisiert die religiöse Frage und mit ihr „die Religion“. Ob das zutrifft, kann allerdings erst ein Längsschnitt zeigen, der die Gesprächsbereitschaften zwischen verschiedenen Themen vergleicht.

Zu Gesprächen mit jedem der drei Partner, aber insbesondere mit kirchlichen Professionellen, motiviert die Kirchenmitgliedschaft weit weniger als die Kirchenbindung. Während Kirchenmitgliedschaft und Kirchenbindung in Deutschland zurückgehen, behält die erste ihren Vorsprung vor der zweiten (Meulemann, 2013, S. 346-347). Die Mitgliedschaft ist Residuum der Säkularisierung ohne besondere Wirkmacht.

Einerlei mit welchem Partner, über 80\% der Deutschen sprechen wenigstens gelegentlich über Religion. Das deutet auf eine starke religiöse Nachfrage (Stark \& Finke, 2000). Sie könnte von den zuständigen Anbietern, den kirchlichen Professionellen, befriedigt werden. Aber die vorliegende Untersuchung hat gezeigt, dass hier die Nachfrage am schwächsten ist. Der natürliche Partikularismus der Familie schlägt den potentiellen Partikularismus der Beziehung zwischen Kirchenvertretern und Gläubigen bei weitem. Können die Anbieter, ohne sich zu überlasten, die Nachfrage steigern? Auf diese Frage gibt die Literatur keine Antwort. Die Untersuchungen zur Rolle des Priesters beschäftigen sich - zumindest auf Seite des Katholizismus (Ziemann, 2007, S. 206-225) - ausschließlich mit Belastungen, widersprüchlichen Erwartungen und Konflikten mit der kirchlichen Hierarchie, also mit Problemen der Anbieter. Weitere Untersuchungen zur Nachfrage, die auch den Gegenstand des Gesprächs erfragen, können vielleicht zur Lösung dieser Probleme beitragen.

\section{Literaturverzeichnis}

Breuer, M. (2012). Religiöser Wandel als Säkularisierungsfolge. Wiesbaden: VS Verlag.

Evangelische Kirche Deutschlands (Hrsg.) (2014). Engagement und Indifferenz. Kirchenmitgliedschaft als soziale Praxis. V. EKD-Erhebung über Kirchenmitgliedschaft. Hannover.

Felling, A., Peters, J. \& Schreuder, O. (1987). Religion im Vergleich: Bundesrepublik Deutschland und Niederlande. Frankfurt: Lang.

Gabriel, O. (2013). Cognitive political engagement. In S. Keil \& O. Gabriel (Hrsg.), Society and Democracy in Europe. London: Routledge. 
Hahn, A. (1974). Religion und der Verlust der Sinngebung. Identitätsprobleme in der modernen Gesellschaft. Frankfurt: Herder \& Herder.

Hermelink, J., Liskowsky, A. \& Grubauer, F. (2014). Kirchliches Personal. In Evangelische Kirche Deutschlands (Hrsg.), Engagement und Indifferenz. Kirchenmitgliedschaft als soziale Praxis. Hannover.

Huber, W., Friedrich, J. \& Steinacker, P. (Hrsg.) (2006). Kirche in der Vielfalt der Lebensbezüge. Die vierte EKD-Erhebung über Kirchenmitgliedschaft. Gütersloh: Gütersloher Verlagshaus.

Karle, I. (2001). Der Pfarrberuf als Profession. Gütersloh: Kaiser.

Krech, V. \& Höhmann, P. (2005). Die Institutionalisierung religiöser Kommunikation. In T. Klatetzki und V. Tacke (Hrsg.), Organisation und Profession. Wiesbaden: VS Verlag.

Nassehi, A. (2009). Religiöse Kommunikation. In Bertelsmann Stiftung (Hrsg.), Woran glaubt die Welt? Analysen und Kommentare zum Religionsmonitor 2008. Gütersloh: Verlag Bertelsmann Stiftung.

Parsons, T. \& Shils, E. (1951). Values, Motive, and Systems of Action. In Dies. (Hrsg.), Towards a General Theory of Action. Theoretical foundations for the Social Sciences. New York: Harper.

Sammet, K. (2006). Religiöse Kommunikation. In W. Huber, J. Friedrich \& P. Steinacker, P. (Hrsg.), Kirche in der Vielfalt der Lebensbezüge. Die vierte EKD-Erhebung über Kirchenmitgliedschaft. Gütersloh: Gütersloher Verlagshaus.

Schützeichel, R. (2010). Kontingenzarbeit. Die psychosoziale Beratung als Funktionsbereich. In M. Ebertz \& R. Schützeichel (Hrsg.), Sinnstiftung als Beruf. Wiesbaden: VS Verlag.

Stark, R. \& Bainbridge, W. (1987). A Theory of Religion. New York: Peter Lang.

Stark, R. \& Finke, R. (2000). Acts of Faith: Explaining the Human Side of Religion. Berkeley: University of California Press.

Tyrell, H., Krech, V. \& Knoblauch, H. (1998). Religiöse Kommunikation. Einleitende Bemerkungen zu einem religionssoziologischen Forschungsprogramm. In Dies. (Hrsg.), Religion als Kommunikation. Würzburg: Ergon.

Ziemann, B. (2007). Katholische Kirche und Sozialwissenschaften 1945-1975. Göttingen: Vandenhoeck \& Ruprecht.

Ziemann, B. (2009). Sozialgeschichte der Religion. Frankfurt: Campus.

Prof. Dr. Heiner Meulemann ist Professor für Soziologie an der Wirtschafts- und Sozialwissenschaftlichen Fakultät der Universität Köln. 